\title{
Towards an SMS-based Social Network for Health Workers in Rural Areas in Myanmar
}

\author{
Joël Vogt, Eugenia Martin \\ Department of Informatics \\ University of Fribourg \\ Fribourg, Switzerland
}

\author{
Edy Portmann \\ Institute of Information Systems \\ University of Bern \\ Bern, Switzerland
}

\author{
Nasim Mahmud \\ Department of Computer Science and Engineering \\ University of Liberal Arts Bangladesh \\ Bangladesh
}

\begin{abstract}
Seventy percent of the population in Myanmar lives in rural areas. Although health workers are adequately trained, they are overburdened due to understaffing and insufficient supplies. Literature confirms that information and communication technologies can extend the reach of healthcare. In this paper, we present an SMS-based social network that aims to help health workers to interact with other medical professionals through topic-based message delivery. Topics describe interests of users and the content of message. A message is delivered by matching message content with user interests. Users describe topics as ICD10 codes, a comprehensive medical taxonomy. In this ICD-10 coded SMS, a set of prearranged codes provides a common language for users to send structured information that fits inside an SMS.
\end{abstract}

\section{Community health workers; Rural health; mHealth; ICD-10}

\section{INTRODUCTION}

After decades of crippling sanctions, Myanmar (also known as Burma) is experiencing a momentum of transformation and economic growth. However, these economic growths predominantly occur in urban areas, especially for former capitals Yangon and Mandalay. Mobile phones and Internet access is available in populated areas. Seventy percent of the total population of Myanmar resides in rural areas, where coverage of mobile phone networks is almost nonexistent [1]. Two foreign companies, telecom operator Ooredoo originating from Qatar, and its Norwegian counterpart, Telenor, have won the bidding for telecom licenses and have the ambitious goal to provide coverage for $75 \%$ to $80 \%$ of the population between 2015 and 2016 [2]. Both companies have pledged investment in this project as part of their social commitment. In rural areas, healthcare coverage is often inadequate. To improve the availability of healthcare, the Health Plan of the Ministry of Health of Myanmar identifies four areas in public health needing to be addressed [1]:

- Need to improve rural health-care coverage.

- Persistence of maternal, infant and child mortality levels that need further reduction.

- Excessive workload of basic health staff.
- Need of quality data for National Health Information Systems.

Our aim in this paper is to develop a mobile question-andanswer system that allows health workers in rural areas to exchange information among their peers as well as medical professionals in other locations via Short Message Service (SMS) and thus facilitate their daily work. In urban areas such as Yangon, medical services are available. However, the medical work force is spread thin in rural areas. The government tries to improve health coverage by posting newly graduated medical doctors to work for two years in rural areas. However, these assignments are often unpopular. While medical professionals in rural areas have adequate training and expertise, they often lack proper equipment [3] [4] [1]

We propose a SMS-based social network, the Mobile Competence Network $(\mathrm{MCN})$, to facilitate communication between health workers in rural areas, medical professionals, and Myanmar's national health service using basic communication technologies. Another challenge is the lack of information about the health situation, especially in remote areas. Using predefined keywords to specify the semantics of the content provides the Ministry of Health with current information about the topics that are being discussed. For example, topics being discussed more frequently could mean that they need more attention.

\section{RELATED WORK}

In this paper, we focus on mobile health (mHealth) for rural areas, in which information and communication technologies are used to extend the reach of healthcare to underserved areas. Even low-tech information and communication technologies, such as mobile phones, can facilitate the promotion of health education and the delivery of basic health care services to previously underserved areas while maintaining economic feasibility [5] [6].

SMSs are popular in mobile health applications for patients as well as for medical professionals. SMS is a part of the GSM (Global System for Mobile Communications) protocol and is 
available on any GSM mobile phone. This makes SMS a widely available, low-cost way to reach a large group. Existing SMS-based mHealth systems have demonstrated the potential of SMS. Examples include data collection (6-8), education. and notification $(9,10)$. SMS-based solutions are designed to send information to an individual user or to broadcast information to a wide user base. SMS-based systems usually provide a client-side application such as JavaME or SIM applications to hide the complexity from users. While this makes it easier for the user to input data, it requires a client-side application to be installed and maintained as the requirements evolve. To avoid client-side applications, users can directly input commands or data fields as SMS text $(6,11)$. This requires a shared language that specifies the terminology, syntax and semantics. The District Health Information System 2 (DHIS2) demonstrated that SMS can be used by medical workers to send semi-structured data using a code system. They found that the limitations of SMS lack of input validation, no visual cues to help users identify the text structure, and the lack of semantics in the definition of codes for the SMS-based interface warrant further investigation (6).

Although medical professionals need to communicate with their peers, the social dimension for community health workers that use SMS is little explored. This is not due to the lack of technical feasibility, as the micro-blogging site Twitter shows. Twitter is a popular social network that allows users to share messages that fit in a single SMS. The content semantics are described using hash tags that can be freely defined.

The contribution of this paper is two-fold:

- A human-readable code-based system based on the widely popular hash tag system found in Twitter that allows users to define their own set of tags for a specific process; and

- A SMS-based social network framework that permits adding social network functionality to existing mHealth systems.

\section{A. SMS-based Mobile Competence Network (MCN)}

The Mobile Competence Network (MCN) is a lightweight, flexible, SMS-based social network system designed to connect community health workers in areas with limited mobile phone network coverage. The strength of the MCN is that it allows users to send structured information in a compact manner (SMS) by using a prearranged set of codes, referred to as codebook, which specify the semantics for a specific set of tasks. A codebook is used by an MCN server that hosts the business logic as well as by the users who compose and receive messages. The codebook replaces the need to install a side application, and thus it is platform-independent.

The business logic of a codebook is encapsulated in an application bundle component that runs on an MCN server. An MCN environment can consist of a network of independent servers. Each server is autonomous, with its applications of bundles instances and database instances. An MCN server manages the application bundle lifecycle and provides an interface to compose, send, and receive SMS messages as well as database access. An application bundle contains data codes and/or action codes. Data codes means that value is either stored or retrieved. A codebook that saves and/or retrieves information during an interaction also specifies a database schema. Action codes allow implementing complex functions that access application programing interfaces (APIs).

The key features of MCN are:

- Lightweight user interface: Users only need a mobile phone with SMS and the codebook to use the MCN system.

- Accommodating existing practices: The codebook, which describes the user interface functionality, can be written in any language and form, for example as a reference sheet that explains each code in Burmese and English. Alternatively, codes can be added to existing documents. It requires minimal training for the users, since users only need a reference for the indicator codes and how to construct a SMS with the code and data value pair.

- Extensibility: The entire functionality that MCN provides is on the MCN server. New features for the users are added by distributing new codebooks and adding the functionality to the MCN server. The server also has a codebook and acts upon the code and data instructions in the SMS.

- Modular design: MCN is designed to extend an existing health information system with a social network functionality. It therefore relies on an external system for user management and data analysis

The MCN system is described in detail in the remainder of this section.

\section{B. System Architecture}

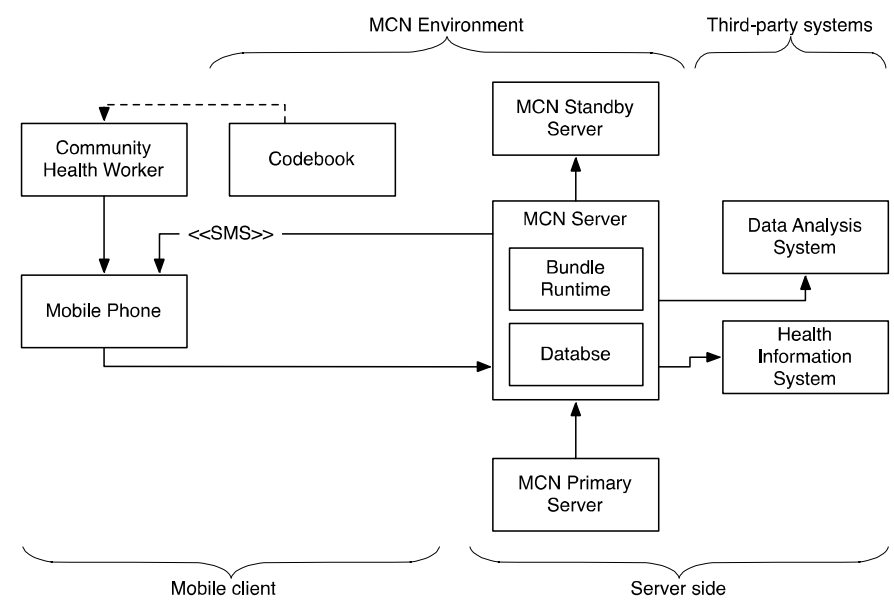

Figure 1 Conceptual architecture of the MCN system

The architectural overview of the MCN system is shown in Fig. 1. The system consists of a mobile and a server subsystem which communicate via SMS. The message content adheres to a communication protocol defined in a codebook.

- The mobile client consists of an ordinary mobile phone that can send and receive SMS, and a codebook that contains the phone number and describes each code for a specific workflow. The user accesses the MCN server by sending and re- 
ceiving messages that adhere to the codes provided in a codebook.

- The server-side consists of one or more MCN servers. A MCN server contains a bundle runtime engine and a database. The bundle runtime engine manages the lifecycle of application bundles that contain the functionality of a codebook. When the server receives a SMS, it parses the content and passes it to the application bundle that is registered for the recipient's number. The database manages the data for the application bundles. In a distributed MCN environment, a primary server replicates its database content to a standby server which has one or more primary servers. A server can assume the role of a primary and standby to build a hierarchy of servers.

MCN follows a component-based software architecture which makes it easy to add functionality by installing a new application bundle and assigning it to a unique phone number. The runtime environment contains the application bundles that contain the functionality of codebooks. The runtime environment manages the lifecycle application bundles and provides interfaces to the database and to SMS communication.

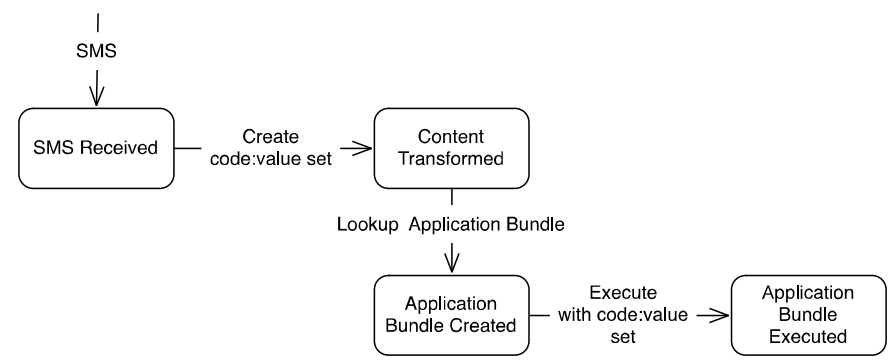

Figure 2 The addressee phone number determines which application bundle is selected.

The state of the runtime environment is shown in Fig. 2. When the MCN server receives a SMS, its content is first converted to a code: data set. The runtime environment contains a table with the unique phone number of each application bundle. When a SMS is received, it looks up the receiver number in the SMS and instantiates and executes the application bundle.

\section{Codebook}

A codebook is a collection of user-defined codes for specific workflow and an application bundle that implements the functionality of each code. We use a component-based design for the following reasons:

- The codebook functionality provided by an $\mathrm{MCN}$ server is extended by installing new application bundles;

- Application bundles can be installed on multiple servers to distribute the load across several servers or provide a fail-safe system; and

- Components are less affected by technological change because it decouples the codebook functionality from concrete implementations.
A code is identified by the \# operator, followed by name of the code that is either an action or a data type. An action with arguments and a data type is followed by a ":" and one or more values separated by "," . Codes are chosen freely and code names have no semantics. The corresponding application bundle on the MCN server has a codebook hashtag map that defines how each code is treated.

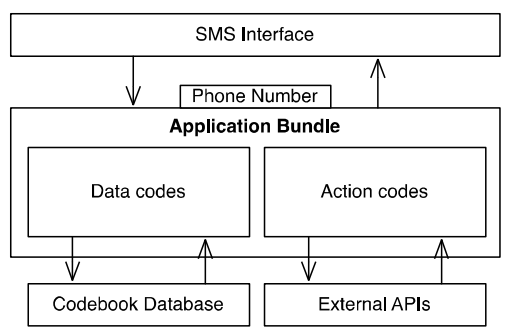

Figure 3 Codebook application bundle

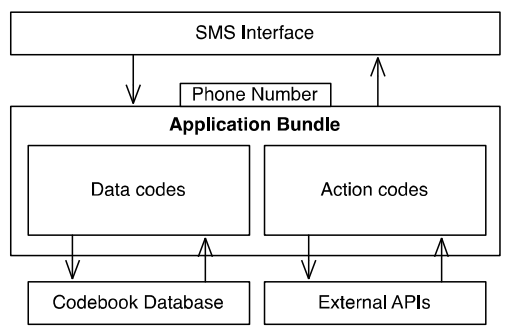

Figure 3 presents a high-level overview of an application bundle. An application bundle defines data codes and/or action codes. Data codes means that value is either stored or retrieved. A codebook that saves and/or retrieves information during an interaction, it also specifies a database schema. Action codes are codes that represent functions that the user wants to execute. Action codes can represent any sort of functionality and access application programing interfaces (APIs). Action codes can also save and retrieve data from the database.

When an application bundle is installed, it is assigned to a phone number. If a database schema has been defined, a new database instance is created according to the database schema of the bundle.

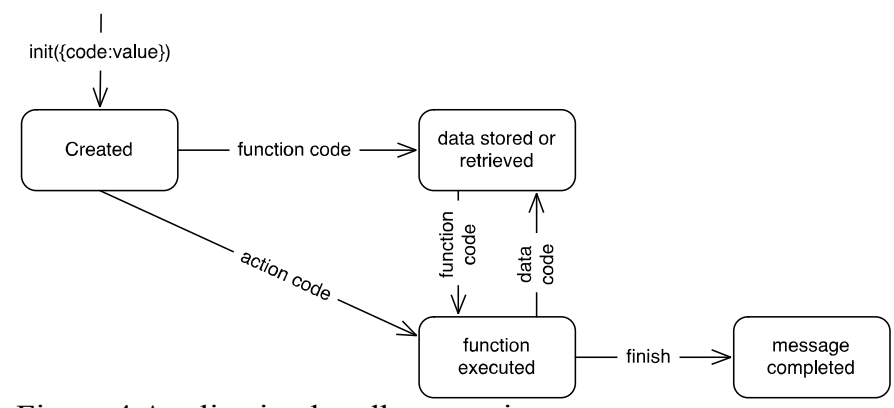

Figure 4 Application bundle execution

The states of an application bundle are shown in Fig. 4. The runtime environment creates the application bundle and passes that run the application bundle with the code: value set passed as argument. The application bundle then stores or retrieves codes that represent data value and executes codes that represent functions. The codes are executed in sequential order, from left to right. The application bundle ends once all the codes have been processed. 


\section{Social Network for disseminating Health-related Messages on ICD-10 Codes}

We present the social network codebook that allows users to send messages to recipients who share similar interests by describing the message content through one or more ICD-10 codes. Users state their interests by registering the ICD-10 codes. Messages are general information that someone wants to share and specific questions or proposals to meet.

We introduce ICD-10 and the advantages of using a welldefined medical taxonomy to specify the content followed by a discussion of the three services this codebook provides.

ICD-10 is the $10^{\text {th }}$ revision of the "International Statistical Classification of Diseases and Related Health Problems," a comprehensive medical classification system created by the World Health organization. ICD-10 was chosen for our system because it is widely used, provides a taxonomy of over 14,4400 structured codes, and uses a concise code notation.

The following example is an extract from ICD-10 ${ }^{1}$ for hepatitis A and two subtypes:

B15 Acute hepatitis A

B15.0 Hepatitis A with hepatic coma

B15.9 Hepatitis A without hepatic coma

The sub- and super-type relationships permit the system to "understand" which topics are related and to include these in the set of relevant topics. For example, a user who is interested in "acute hepatitis A in general" registers with the code B15. Because of the well-defined classification, the social network module will also send messages tagged with the B15.0 and B15.9 to that user, because both are subtypes of B15.

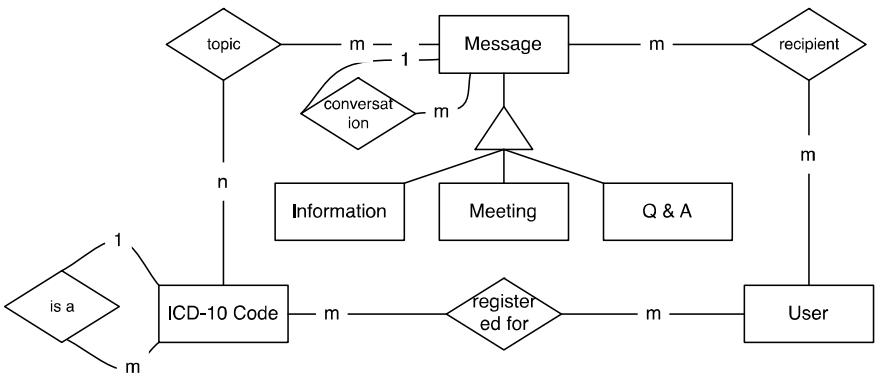

Figure 5 Social network data model

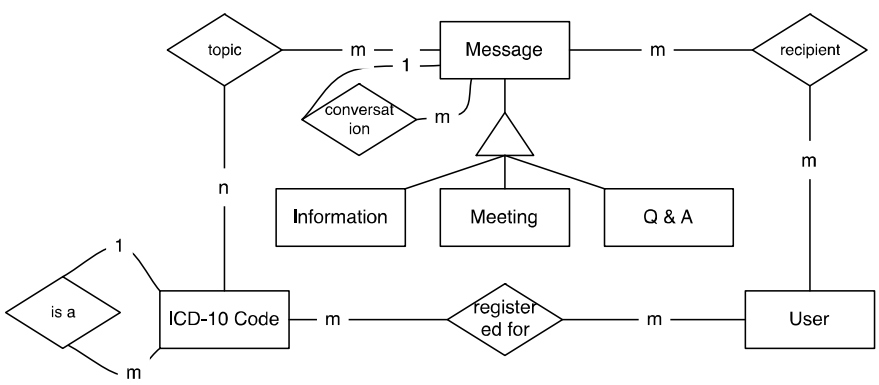

Figure 5 shows the data model for the social network. A user specifies the topics of interest by registering ICD-10 codes.
This relationship specifies the sub- and super-class relationship between codes. When a message arrives, the system determines the recipients by querying the users that registered for the ICD-10 codes (and their sub codes) that the sender specified in the message. The system stores the codes for each message. It also explicitly stores the recipients of each message, because users' topics can change over time. Messages that are replies to previous messages, for example to answer a question or participate in a group discussion, are stored as conversations. The entire data store for the social network codebook is stored in a PostgreSQL relational database. Codes that are commands are stored in a Python dictionary with the corresponding method as a value.

The social network codebook is as follows:

\section{General commands \\ Code Description}

\#icd10: $\quad$ List of ICD-10 codes to specify the content of

\#call: the message

call: $\quad$ Command to return phone number and the name of the sender by specifying the message ID number.

\#register Command to state subject of interest, followed by \#icd10. Example: \#register\#icd10: B15,A90

\#unregister Command to remove subject, followed by \#icd10. Example: \#unregister\#icd10: B15

\#msg: $\quad$ The message content written by the user. Example \#msg: some text

\section{Meeting \\ Code \\ Description}

\#new

\#par:

\#close:

Command to propose a new meeting, followed by \#icd10 and \#msg. Example: \#new\#icd10: A90\#msg: Buy mosquito nets and DEET Command to confirm participation to meeting by replying with the meeting ID. The meeting ID is added to the SMS. Example: \#par: 42 notify participants. The user who proposes the meeting also receives the auto-generated ID per SMS.

Question and Answers

\section{Code Description}

\#q Command to ask a question, followed by \#icd10 to specify the subject of the question and \#msg with the question

\#a: $\quad$ Command to reply to a question, followed by \#msg with the answer. Each question has an auto-generated number to answer

\section{Question and Answers}

\section{Code Description}

\#inf Command to broadcast information to a group for user without expecting reply. Command is followed by \#icd10 to specify the subject of the question and \#msg with the question

\footnotetext{
${ }^{1} \quad{ }^{1}$ http://www.who.int/classifications/icd/en/
} 


\section{E. MCN as Extension of DHIS2}

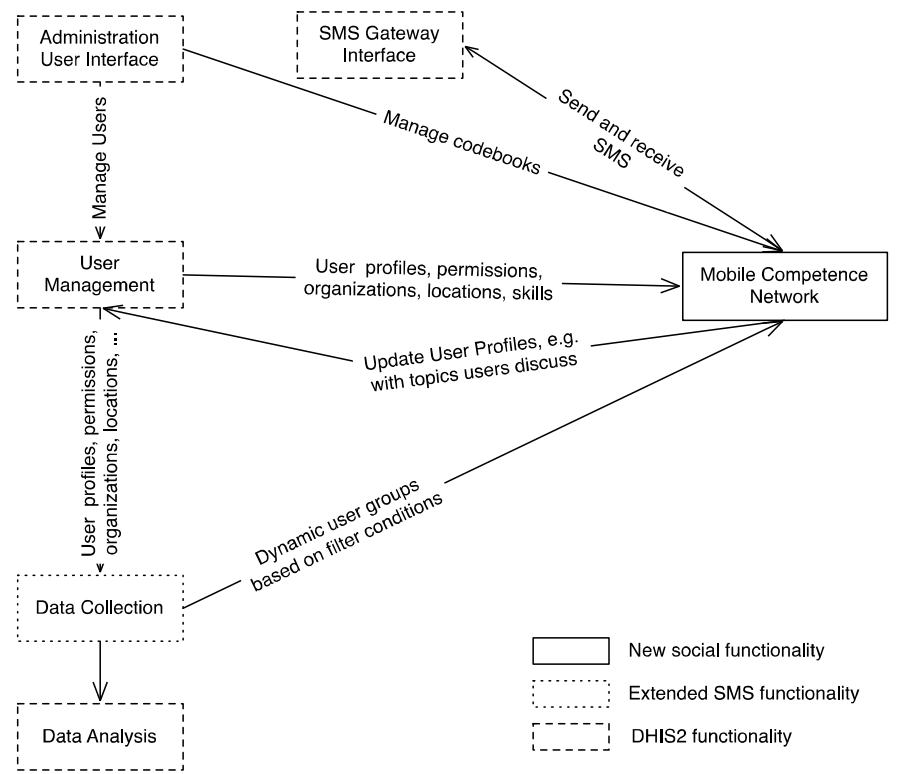

Figure 6 Adding social network functionality to DHI2

$\mathrm{MCN}$ is designed to add an mHealth extension to the existing health information system program. A notable example is the DHIS2 developed at the University of Oslo, Norway. We use DHIS2 as an example of how MCN can add social network functionality to an existing health information system.

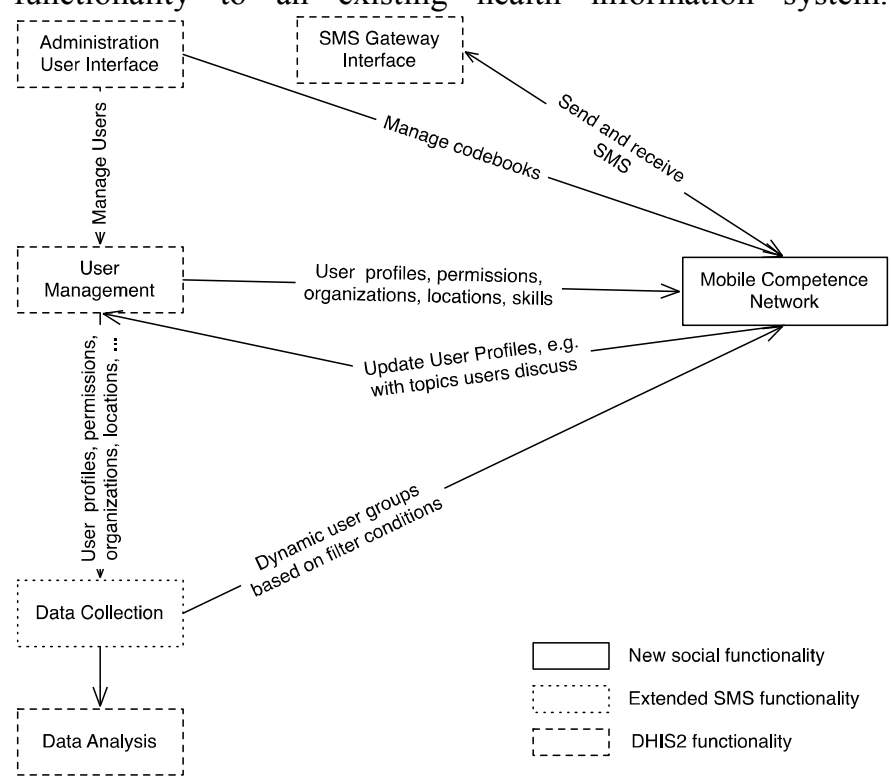

Figure 6 shows how conceptually the mobile competence module adds the social network extension to DHIS2. The services that $\mathrm{MCN}$ provides are to orchestrate the message exchange between users. The MCN module decides how to react to a message based on the codes in the SMS, the people in the network, and context of the sender and the recipients. The code semantics are specified with the administration user interface of DHIS2 and provided to the users on paper. In addition, a corresponding codebook application bundle is installed in the MCN module to implement each code. The user context consists of the static and dynamic context. The static context is the user role, organization, skillset, and selected topics of interest. The dynamic context is the user's location, the time and information inferred from the user's personal messages, as well as from the entire message pool.

\section{USE CASE: SMS-BASEd HEALTH SOcIAL NETWORK}

In this section, we propose how the social network module discussed in the previous section could help community health workers who are geographically apart, organize meetings, ask questions of their peers and medical professionals outside their region, or share information. $\mathrm{MCN}$ assists users by managing information flow based on the topics specified in the message and tracking the state of conversations. We first give an overview of how the healthcare system in rural areas in Myanmar will motivate the use of an SMS-based social network to help medical professionals to contact their peers and organize conferences or meetings.

The administrative division of interest for rural health is a township. In Myanmar, a township is a third-level administrative division with a population of 100,000 to 300,000 . A township health system forms the backbone of the primary and secondary health for the population and also monitors the township health situation, such as malaria outbreaks. The overall responsibility at the township level lies with the township medical officer, who is a trained medical doctor. A township has three to five rural health centers, one for each four or five sub-centers. A rural health center employs up to 13 medical professionals and support staff who care for a population of 20 , 000 to 25,000 . For villages that cannot be frequently visited by midwives, community health workers and auxiliary health workers provide basic coverage. These people are volunteers who have been trained by the township health authority (3).

In this use case, the township medical officer suggests setting up a social network to facilitate daily tasks of health workers by making it easier to access and share information. The township has GSM coverage in populated areas and most health workers own a mobile phone. To use the existing infrastructure, the township medial officer accesses a MCN system installed on a server at the township hospital and connected to a SMS gateway. The social network functionality previously described is installed. In addition, each health worker is given a printed copy of the codebook with the code descriptions translated to Burmese as well as a subset of the ICD-10 taxonomy with the topics relevant for that region.

Sharing information: A community health worker notices an increasing number of dengue cases in her community. She shares this information with her colleagues by sending the following SMS \#icd: A90\#msg: More cases of dengue. The message is delivered to all users that subscribed to A90 or a subtype.

Question and Answers: A community health worker has a patient with high fever. He suspects dengue but prefers to consult a medical doctor. \#icd: A90\#q\#msg: Suspect A90, atypical symptoms, please advise. A specialist in Yangon sees the question (ID 23). She suspects dengue and wants to call the community health worker. She replies with: \#a: 23\#msg: will 
call\#call. She receives the number of the community health worker who sent the message and calls him.

Arrange meetings: The township medical officer receives a notice from the Ministry of Health concerning a new shipment of bed nets that will be delivered. She arranges a meeting to discuss how many nets are needed for the township. \#icd: B54\#new\#msg: discuss shipment of bed nets, next week at hospital.

\section{CONCLUSION AND OUTLOOK}

In this paper, we proposed a SMS-based social network application for health workers in rural areas. The system makes use of available low-cost technologies already available or are deemed affordable. We chose SMS for two reasons: it poses no additional requirements for mobile networks or mobile phones, as SMS is part of GSM. Users need a mobile phone and a list of codes that replaces the mobile client application and acts as a communication protocol. The application does not require processing power on the mobile phone and thus energy, as all the computations are executed on the server. Furthermore, SMS are less affected by disruptions than data networks and is available when the signal strength is weak. The codebook approach makes it easy to translate user interfaces using existing tools such as a word processor or pen and paper.

We are currently preparing the implementation of this application for rural areas in Myanmar. MCN will be integrated into the existing DHIS2 to integrate the mobile workface with the health information system at the township hospital and clinics.

\section{ACKNOWLEDGMENT}

The authors express their utmost gratitude to Dr. Nilar Tin for her kindness and providing us with invaluable insight into the health system in Myanmar.

\section{REFERENCES}

[1] WHO Country Cooperation Strategy 2008-2011, Myanmar., 2008.

[2] Myanmar Delays Awarding Telecom Licences - WSJ.com.

[3] N. Tin, Township Health System in Myanmar.

[4] N. Tin, S. Lwin, N. N. Kyaing, T. T. Htay, J. Grundy, M. Skold, T. O'Connell and S. Nirupam, "An approach to health system strengthening in the Union of Myanmar.," Health Policy, vol. 95, no. 2-3, pp. 95-102, 5 2010 .

[5] A. B. Labrique, L. Vasudevan, E. Kochi, R. Fabricant and G. Mehl, "mHealth innovations as health system strengthening tools: 12 common applications and a visual framework," Global Health: Science and Practice, vol. 1, no. 2, pp. 160-171, 2013.

[6] S. Mishra and I. P. Singh, "mHealth: A developing country perspective," Making the e-health connection, pp. 1-9, 2008.

[7] T. A. Sanner, L. K. Roland and K. Braa, "From pilot to scale: Towards an mHealth typology for low-resource contexts," Health Policy and Technology, vol. 1, no. 3, pp. 155-164, 92012.

[8] P. Petrucka, S. Bassendowski, H. Roberts and T. James, "mHealth: A Vital Link for Ubiquitous Health," Online Journal of Nursing Informatics (OJNI), vol. 17, no. 2, 2013.

[9] A. Chib, H. Wilkin and B. Hoefman, "Vulnerabilities in mHealth implementation: a Ugandan HIV/AIDS SMS campaign," Global health promotion, vol. 20, no. 1 suppl, pp. 26-32, 2013.

[10] H. Chhor, R. Dobbs, D. Nguyen Hansen, F. Thompson, N. Shah and L. Streiff, Myanmar's moment: Unique opportunities, major challenges, 2013.

[11] J. Barrington, O. Wereko-Brobby, P. Ward, W. Mwafongo and S. Kungulwe, "SMS for Life: a pilot project to improve anti-malarial drug supply management in rural Tanzania using standard technology.," Malar J, vol. 9, p. 298, 2010.

[12] Increasing Access to Health Workers in Remote and Rural Areas Through Improved Retention: Global Policy Recommendations, World Health Organization, 2010, p. 72.

[13] Improving maternal and newborn access to services in Ghana, World Health Organization, 2013. 